\title{
A Rare Case: Genetically Confirmed Newborn with Thanatophoric Dysplasia Type 1 (TD1)
}

\author{
I Gusti Ayu Dwi Aryani ${ }^{1, ~ *, ~ I ~ M a d e ~ A r i m b a w a ~}{ }^{1}$, Made Kardana ${ }^{1}$, Ni Nyoman Ayu Dewi ${ }^{2}$, \\ Pande Putu Yuli Anandasari ${ }^{3}$
}

${ }^{1}$ Department of Child Health, Medical Faculty of Udayana University, Sanglah Hospital, Denpasar, Indonesia

${ }^{2}$ Department of Biochemistry, Medical Faculty of Udayana University, Denpasar, Indonesia

${ }^{3}$ Department of Radiology, Medical Faculty of Udayana University, Sanglah Hospital, Denpasar, Indonesia

Email address:

dwiaryani51@gmail.com (I G. A. D. Aryani)

${ }^{*}$ Corresponding author

\section{To cite this article:}

I Gusti Ayu Dwi Aryani, I Made Arimbawa, Made Kardana, Ni Nyoman Ayu Dewi, Pande Putu Yuli Anandasari. A Rare Case: Genetically Confirmed Newborn with Thanatophoric Dysplasia Type 1 (TD1). International Journal of Genetics and Genomics.

Vol. 9, No. 1, 2021, pp. 1-5. doi: 10.11648/j.ijgg.20210901.11

Received: December 20, 2020; Accepted: January 4, 2021; Published: January 12, 2021

\begin{abstract}
Thanatophoric dysplasia (TD) is the most frequent sporadic lethal skeletal dysplasia with an incidence about 1 in $17.000-50.000$ births. Diagnosis of TD can made at prenatal period by ultrasound and immediately after birth based on clinical examination, radiologic studies, histopathology and molecular analysis. Thanatophoric dysplasia is subdivided in two dinstinct phenotypes: 1) Thanatophoric dysplasia type 1 (TD1) is characterized by curved "telephone receiver" femora and mild craniosynostosis or 2) Thanatophoric dysplasia type 2 (TD2) includes straight femora and cloverleaf skull. Girl baby was born from 44 years old female with 35th weeks of gestation (WOG) with non-consanguineous marriage. The patient looked lethargic accompanied with rapid breathing with chest indrawing. On examination the patient looked dysmorphic, large head with frontal bossing without clover leaf skull, upper and lower limbs were extremely short. Babygram showed head was large, thoracic cavity was small and narrow, the ribs were short, and "telephone receiver" like curved femora was noted. Mutational analysis confirmed a heterozygous allele p.Tyr373Cys mutation in the Fibroblast Growth Factor Receptor 3 (FGFR3) gene. The patient was diagnosed with TD1. The general condition and respiratory distress did not improve and the patient was died on the sixteenth days of hospitalization. Thanatophoric dysplasia type 1 was rare disease and lethal case. The diagnosis based on clinical examination, radiologic studies, histopathology and molecular analysis. Lethality in thanatophoric dysplasia is caused mainly by respiratory distress due to a narrow thorax.
\end{abstract}

Keywords: Thanatophoric Dysplasia Type 1, Telephone Receiver, FGFR3

\section{Introduction}

Thanatophoric dwarfism was first described in 1967 by Maroteaux and colleagues. In 1977, the term thanatophoric dwarfism was changed to thanatophoric dysplasia (TD) $[1,2]$. The name was derived from the Greek word thanatos meaning 'death' and phoros meaning 'provoking' [2]. Thanatophoric dysplasia is the most frequent sporadic lethal skeletal dysplasia with an incidence about 1 in 17.000 50.000 births. The prevalence of TD was $2.1-3$ per 100.000 live births in the United States of America (USA), 5 per 100.000 live births in Latin America, and 2.4 per 100.000 live births in West Scotland. The other study reported low TD prevalence rate of 0.29 per 100.000 births in Japan [1, 3-6].

Thanatophoric dysplasia is traditionally divided into two forms were TD1 and TD2 on a basis of the radiographic appearances. The two subtypes can be differentiated by the skull shape and femur morphology. Thanatophoric dysplasia type 1 is characterized by radiological features such as shortness and bowing in the long bones, hypoplasia in the vertebral corpus and in some cases a cloverleaf like appearance on the skull. While the shortness of the bones in TD2 is not as significant as in TD1 and there is no bowing in the long bones. In almost all TD2 cases, the skull has a 
cloverleaf like appearance, related to the early closure of the coronal and lambdoid sutures and the expansion of the temporal lobe. In TD1, the femur is curved like a "telephone receiver", while in TD2 the femur is straight and the cloverleaf skull deformity is significant. Thanatophoric dysplasia type 1 being the most common subtype $[5,7,8]$

Skeletal dysplasia can diagnosed at the prenatal period. Thanatophoric dysplasia is a lethal disorder of fetus and can be detected by ultrasound done in second trimester. Accurate prenatal diagnosis of skeletal dysplasias still presents a considerable clinical challenge due to phenotypic variability with overlapping features [3]. Thanatophoric dysplasia is caused by a mutation of the Fibroblast Growth Factor Receptor 3 (FGFR3). This results in constitutive activation of the FGFR3 tyrosine kinase and leads to perturbation of processes in bone formation and cerebral cortical development [1]. Thanatophoric dysplasia has been considered to be caused by a dominant mutation and the recurrence risk was low $[1,3,9]$.

Early diagnosis helps in prognostication and termination can be offered to the patient with TD. Lethality of a particular diagnosis is mainly related to the associated pulmonary hypoplasia from an abnormally formed restrictive thorax. There are some recent reports of survival with aggressive perinatal management in infants previously considered to have TD. These infants frequently have severe growth retardation and chronic respiratory insufficiency and some have mental retardation either secondary to underlying central nervous system anomalies or as a result of chronic respiratory insufficiency $[1,3,9-11]$. The objective of this case series is to describe the clinical examination, radiologic studies, and molecular analysis of TD1.

\section{Case Illustration}

Girl baby was born from 44 years old female with $35^{\text {th }}$ weeks of gestation (WOG) with non - consanguineous marriage, had normal regular antenatal check-ups and no chronic illnesses or family history of congenital abnormality. There were no history of fever and urinary tract infection during pregnancy. There were no history of rashes, drug intake and radiation exposure during this pregnancy. Mother was a nonsmoker and a non-alcoholic. She routinely consumed multivitamins during pregnancy given by the obstetrician. There was the third pregnancy. The mother has history of spontaneous abortion of 2 months period of gestation at the second pregnancy and no investigations were done after the abortion. The details regarding any other associated anomalies in the previous pregnancy are not known.

At $29^{\text {th }}$ WOG, the mother came to regular antenatal check-up, 4D ultrasound was done revealed average age of fetus as $24^{\text {th }}$ WOG, estimated fetal weight was 1529 grams, biparietal diameter (BPD) corresponding to $29^{\text {th }}$ WOG, head circumference $(\mathrm{HC})$ corresponding to $30^{\text {th }} \mathrm{WOG}$, abdominal circumference corresponding to $31^{\text {st }}$ WOG, humerus length corresponding to $16^{\text {th }}$ WOG only, and femoral length corresponding to $14^{\text {th }}$ WOG only. Fetal skeletal morphology revealed small thorax, frontal boosing and spondylosis. All four limbs were small to corresponding gestation (micromelia) which is suggestive of thanatophoric dysplasia. The obstetrician counselled regarding termination of pregnancy owing to poor fetal prognosis but the parents wished to continue the pregnancy. At $35^{\text {th }}$ weeks plus gestational age, the patient was delivered by sectio caesarea due to premature rupture of membranes and polyhydramnios.

The patient did not cry immediately after birth. She looked lethargic accompanied with rapid breathing with chest indrawing. On examination the patient looked dysmorphic with skeletal dwarfism. Face was oedematous, large head with frontal bossing without clover leaf skull, depressed nasal bridge, eyes looked widely spaced, low set ears and the neck was shortened. Upper and lower limbs were extremely short with short stubby fingers and deep skin creases. There was relative narrowing of the thorax with distended abdomen. Furthermore, abduction of bilateral hip joint was seen (Figure 1). After stabilized the patient admitted to level II neonatal ward with incubator and continuous positive airway pressure (CPAP).

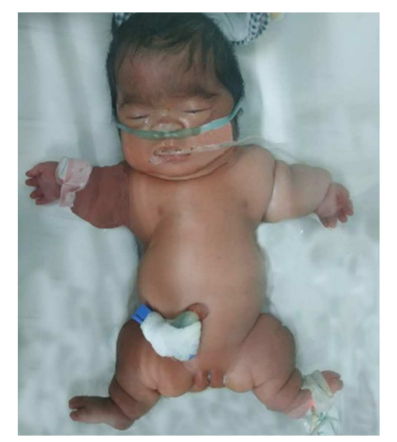

Figure 1. Neonate with frontal bossing, depressed nasal bridge, short neck, low set ears, narrow chest, protuberant abdomen, short extremity, and deep skin creases.

Patient birth weight was 2290 grams (25th-50th percentile of Lubchenco curve), birth length was $34 \mathrm{~cm}(<10$ th percentile of Lubchenco curve), and head circumference was $33 \mathrm{~cm}$ (50th-75th percentile of Lubchenco curve). All the limbs were small with deformed attitude (Upper segment: $23 \mathrm{~cm}$ and lower segment: $11 \mathrm{~cm}$, and US/LS ratio was 2.09).

On the first day of hospitalization patient was shortness of breath. Physical examination revealed decreased activity, tone, and reflex, temperatures ranged between $36.5-36.9^{\circ} \mathrm{C}$, heart rate $145-170$ beats/minute, respiratory rate $60-70$ beats/minute. No anemic, no icteric, round pupils, and good light reflexes. Lung and cardiac auscultation revealed grunting, mild retraction and no heart murmur. Complete blood count revealed leukocyte $11.21 \times 103 / \mu \mathrm{L}$, neutrophils $6.69 \times 103 / \mu \mathrm{L}$ (59.69\%), hemoglobin $18.33 \mathrm{~g} / \mathrm{dL}$, hematocrit $57.63 \%$, platelets $161.10 \times 103 / \mu \mathrm{L}$, blood sugar $65 \mathrm{mg} / \mathrm{dL}$, IT ratio 0.09 , and blood smear revealed no toxic granule or vacuolization.

Immediate postnatal babygram showed head was large without clover leaf shaped. Thoracic cavity was small and narrow, the ribs were short and horizontally placed. Bilateral humerus and femori were extremely short with metaphyseal 
flaring. Other bones like radius, ulna, tibia and fibula were also very short, the vertebral bodies showed flattened (platyspondyly), pelvis was narrow and small. Typical features such as "telephone receiver" like curved femora were noted (Figure 2). Cranial sonography showed normal structural of the brain, without craniosynostosis.

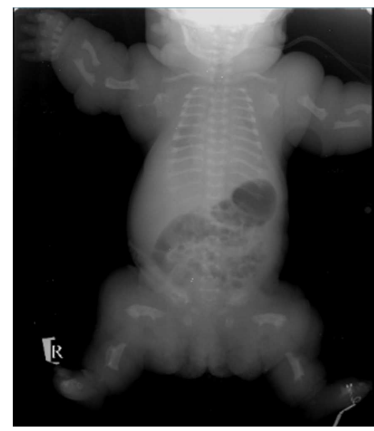

Figure 2. Babygram showed small thoracic cavity, platyspondyly, short humerus, femori, and forearm bones, short bowed femurs giving "telephone receiver" appearance.

Patient was assessed with preterm infant (35 weeks), low birth weight (2290 grams), appropriate for gestational age, suspect early-onset sepsis. With facial features and skeletal abnormalities the diagnosis of TD1 was made. Patient was treated in level II neonatal ward with incubator, CPAP support, total parenteral nutrition, and first line intravenous antibiotics (ampicillin $50 \mathrm{mg} / \mathrm{kg} /$ dose every 12 hours and amikacin 7.5 $\mathrm{mg} / \mathrm{kg} /$ dose every 24 hours). Blood culture was taken before antibiotic administered. Monitoring was performed for symptoms, vital sign, and fluid balance. Patient was planned for molecular analysis.

On the fifth days of hospitalization respiratory distress had not improved, still lethargy and no temperature instability. The patient look icteric with Kramer IV. Bilirubin test revealed total bilirubin $17.61 \mathrm{mg} / \mathrm{dL}$, direct bilirubin $0.25 \mathrm{mg} / \mathrm{dL}$, and indirect bilirubin $17.36 \mathrm{mg} / \mathrm{dL}$. Thyroid function test revealed thyroid stimulating hormone (TSH) $3.98 \mathrm{IU} / \mathrm{mL}$ and free thyroxine (FT4) $1.78 \mathrm{ng} / \mathrm{dL}$. Phototherapy was performed for three days.

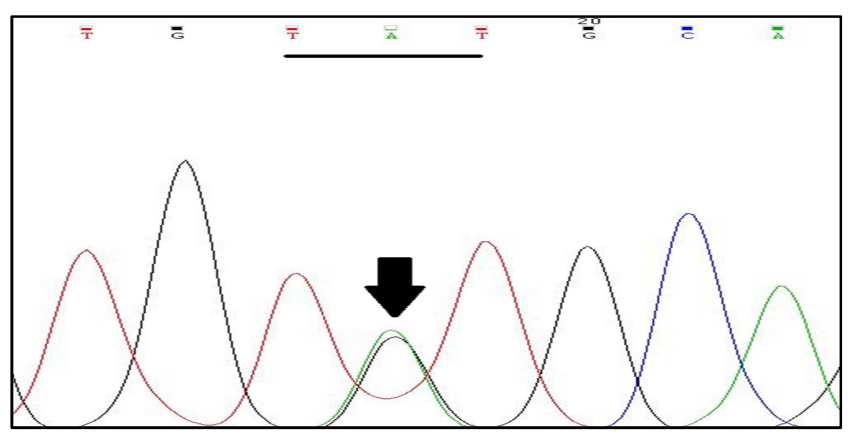

Figure 3. Mutation analysis confirmed a heterozygous allele p.Tyr373Cys mutation in the FGFR3 gene.

Evaluation of complete blood count on tenth days of hospitalization revealed leukocyte 14.54 x $103 / \mu \mathrm{L}$, neutrophils $7.39 \times 103 / \mu \mathrm{L}(50.79 \%)$, hemoglobin $16.85 \mathrm{~g} / \mathrm{dL}$, hematocrit $51.55 \%$, platetets $245.80 \times 103 / \mu \mathrm{L}$, IT ratio 0.14 , and procalcitonin $0.11 \mathrm{ng} / \mathrm{mL}$. Bilirubin test after phototherapy revealed total bilirubin $7.24 \mathrm{mg} / \mathrm{dL}$, direct bilirubin $0.55 \mathrm{mg} / \mathrm{dL}$, and indirect bilirubin $6.69 \mathrm{mg} / \mathrm{dL}$. Blood culture revealed was no growth. Antibiotic was stopped.

Molecular analysis of the FGFR3 gene was done after birth and was recently confirmed in a clinical laboratory. The patient screened for mutation foci in exon 7 (R248C, S249C) and exon 10 (G370C, Y373C). Mutational analysis confirmed a heterozygous allele p.Tyr373Cys mutation in the FGFR3 gene (Figure 3 ). This is a de novo missense mutation that resulted in a change in amino acid code from tyrosine to cysteine at codon 373 (TAT $\rightarrow$ TGT) in the juxta extramembrane domain of the receptor (Y373C).

Baby was managed with CPAP and other supportive therapy as per unit protocol. However, the general condition and respiratory distress did not improved and baby was died on the sixteenth days of hospitalization caused mainly by respiratory distress to a narrow thorax.

\section{Discussion}

Thanatophoric dysplasia is the most common neonatal lethal skeletal dysplasia. Diagnosis of TD can made at prenatal period by ultrasound and immediately after birth based on clinical examination, radiologic studies, histopathology and molecular analysis. Prenatal diagnosis of skeletal dysplasias is initially accessed by two-dimensional sonography (2D-US) during the $2^{\text {nd }}$ trimester where fetal biometry and fetal anatomy including skeleton and other systems as head and thorax are examined in detail. Ultrasound is the primary imaging modality used for the initial diagnostic evaluation of an affected fetus and the diagnostic accuracy has been reported to be $31-65 \%$ depending on type of skeletal dysplasia. Skeletal disorders are first suspected during routine sonographic examination after a shortened long bone or other abnormal skeletal finding. Femur length measurement is still considered the best parameter in the evaluation of skeletal dysplasias [3, 10, 12]. Fetus showing femora or humeri length $<5^{\text {th }}$ centile or $-2 \mathrm{SD}$ during the $2^{\text {nd }}$ trimester $(<24$ weeks) should be reevaluated by expert ultrasonographer and genetic counseling should be offered. In this case, mother had routine sonographic examination. At $29^{\text {th }}$ WOG sonographic examination revealed shortened long bone then reevaluated by expert ultrasonographer. Humerus length corresponding to $16^{\text {th }}$ WOG only, and femoral length corresponding to $14^{\text {th }}$ WOG only.

Shape and size of the head are other parameters of critical importance that contribute in the identification of abnormal cases. In a prospective study, suggested that one characteristic finding in case of TD1 was relative macrocephaly ( $\mathrm{HC}>90^{\text {th }}$ centile). Etiology of macrocephaly in TD1 cases observed in ultrasound can be associated with the role of FGFR3 activation in selective growth and expansion of occipitotemporal cortex. In one cohort study, 10/16 cases thanatophoric dysplasias presented with BPD and/or $\mathrm{HC}>90^{\text {th }}$ centile. Biparietal diameter was $>95^{\text {th }}$ centile in $2 / 3$ TD1 cases and on the $90^{\text {th }}$ 
centile in $1 / 3$ TD1 cases. Head circumference was $>95^{\text {th }}$ centile in $1 / 3$ TD 1 cases and on the $90^{\text {th }}$ centile in $2 / 3$ TD 1 cases. Polyhydramnios also is frequently associated with TD1 $[3,5]$. In this case, the prenatal ultrasound at $29^{\text {th }}$ WOG revealed no macrocephaly with BPD corresponding to $29^{\text {th }}$ WOG and HC was corresponding to $30^{\text {th }}$ WOG. At $35^{\text {th }}$ weeks plus gestational age, the patient was delivered by sectio caesarea due to premature rupture of membranes and polyhydramnios.

Thanatophoric dysplasia is a lethal disorder of fetus and can be detected by ultrasound done in second trimester. Early diagnosis helps in prognostication and termination can be offered to the patient. However hence, if the condition is diagnosed prenatally couples should receive genetic counseling and advised for termination of pregnancy. If the diagnosis is made after the birth of the baby, management is solely supportive, as death occurs from respiratory insufficiency within hours to days. In a retrospective study, termination was reported to be done in $37.7 \%(23 / 61)$ of TD fetuses [1, 11]. In this case, prenatal ultrasound result was suspect TD with differential diagnosis achondroplasia and the obstetrician suggest to termination the fetus, but the parents were refused.

At birth, TD is characterized by shortening of the limbs, narrowing thorax, platyspondyly and macrocephaly. Thanatophoric dysplasia is traditionally divided into two forms on a basis of the radiographic appearances. The two subtypes can be differentiated by the skull shape and femur morphology. Thanatophoric dysplasia type I, the most common subtype, characterized by curved and short femur which is in a "telephone receiver" like configuration and no cloverleaf shaped skull. Also, the abdomen appears protuberant in comparison with the chest which is narrow and small. The fetuses with TD2 are reported to have cloverleaf skull which means a trilobed skull and straight femora $[5,7]$. In this case, patient birth length was $34 \mathrm{~cm}\left(<10^{\text {th }}\right.$ percentile of Lubchenco curve), all the limbs were extremely short with deep skin creases, without macrocephaly, no cloverleaf skull and protuberant abdomen. The radiologic examination showed thoracic cavity was small and narrow, the ribs were short, and short bowed femurs giving "telephone receiver" appearance.

However, differentiation from other forms of short limb dwarfism, such as achondroplasia and the diastrophic dysplasia family of osteochondrodysplasia, can be difficult. In TD1, shortening of the long bones, the appearance of the skull, and the presence of spinal curvature and narrowing of the spinal canal have a similar configuration to that of achondroplasia. In TD1 the vertebral bodies are very flat and underdeveloped, whereas in the typical form of achondroplasia, the vertebral bodies are square and cuboid. However, the most important difference between the entities is that TD1 is characterized by severe shortening of the ribs. Ribs are thinner and shorter than normal, this reduces the volume of the thorax and causes lung hypoplasia, restricted lung volume, and severe respiratory distress that leads to death within a few hours of birth $[1,3]$. In this case, patient had respiratory failure due to small and narrow thoracic cavity can be differentiation from the others form of skeletal dysplasias. With facial features and skeletal abnormalities the diagnosis of
TD1 was made.

Molecular analysis can be a great complementary tool to confirm or differentiate the suspicions based on the radiological and morphological features of skeletal dysplasias. Thanatophoric dysplasia is caused by mutation of the FGFR3 gene, which is located on the short arm of chromosome 4 . This activation of FGFR3 results in increased proliferation and decreased apoptosis $[5,15]$. Fibroblast growth factor receptor 3 gene is a membrane bound receptor protein, which consists of an extracellular immunoglobulin like domains, transmembrane domain, and intracellular tyrosine kinase (TK) domains. When it binds to ligands such as fibroblast growth factors, lateral dimerization of the receptor occurs, an activation of TK follows and the signal transduction proceeds. In normal states, FGFR3 regulates proliferation, differentiation and development of cell in the bone and cerebral cortex. A gain of function mutation of FGFR3 makes TK activated independently, which imposes various developmental defects. Pathomechanism of TD is a constitutional activation of FGFR3 in the cartilage by stable dimer formation promotes its translocation into the nucleus, where it interferes with terminal differentiation of chondrocyte. For TD1, heterogeneous mutations alter an extracellular amino acid to cysteine, such as R248C, S249C and $\mathrm{Y} 373 \mathrm{C}$. On the other hand, TD2 results from a $\mathrm{K} 650 \mathrm{E}$ substitution in FGFR3 [1, 3, 7, 13]. In 2016, one study showed that $90 \%$ of TD1 mutations were either pArg $248 \mathrm{Cys}(66 \%)$ or pTyr373Cys (24\%). Cases with mutation at Y373C had more severe radiological manifestations than TD1 with R248C, but there was phenotypic overlap [2]. During the last few years, molecular analysis has been used for a definite diagnosis in utero. In this context, diagnosis of FGFR3-related skeletal dysplasias suspected upon specific signs in prenatal ultrasonography can be performed in a molecular genetic diagnostics laboratory [3]. In this case, molecular analysis performed from the blood sample, patient screened for mutation foci in exon 7 and exon 10 of FGFR3 gene. Molecular analysis confirmed a mutation Y373C of exon 10 (Tyr373Cys) in the FGFR3 gene.

Most of the fetuses with TD1 die in utero. Of the few fetuses which are born alive, succumb in the early neonatal period. Unassisted neonates usually die within hours or days due to respiratory insufficiency. Lethality in skeletal dysplasias is caused mainly by pulmonary hypoplasia secondary to a narrow thorax. Narrow and "bell shaped" thorax rather than extremely small thoracic circumference was the prominent characteristic in the TD1 [3]. Infrequently cervical cord compression at a narrowed foramen magnum associated with posterior arch anomaly may be the cause of death $[4,11]$. One case reported a male age nine years with TD1. He required tracheostomy and ventilatory support. At age three years, he demonstrated stable ventriculomegaly, craniosynostosis, and little limb growth. By age eight years, he had seizures, bilateral hearing loss, kyphosis, and both joint hypermobility and joint contractures. At age nine years, the limbs had grown little. He was severely developmentally delayed and had no language. Final height was estimated to be 
$80-90 \mathrm{~cm}$. In the other case, one patient with TD1 can survive until 28 years old managed her own respiration by ventilation support [14]. In this case, patient had severe respiratory distress and was managed by CPAP and other supportive therapy. The general condition and respiratory distress did not improve and baby was died on the sixteenth days of hospitalization.

Without proper genetic counselling, most families had a fetus with thanatophoric dysplasia would be too worried to have further pregnancy. Since majority of the cases of TD occur sporadically, parents with only one previously affected fetus the recurrence risk in subsequent pregnancies is low. A general empiric recurrence risk for TD1 is estimated as 2-5\% $[4,10]$. To relieve parental anxiety, prenatal ultrasound examination may be offered in subsequent pregnancies to identify features suggestive of TD [7].

\section{Conclusions}

In Girl baby was born by sectio caesarea from healthy and non-consanguineous parents with $35^{\text {th }}$ weeks of gestational age. She did not cry immediately after birth. She looked lethargic accompanied with rapid breathing with chest indrawing. On examination the patient looked dysmorphic with skeletal dwarfism. Face was oedematous, large head with frontal bossing without clover leaf skull, depressed nasal bridge, eyes looked widely spaced, low set ears and the neck was shortened. Upper and lower limbs were extremely short. Babygram showed head was large without clover leaf shaped. Thoracic cavity was small and narrow, the ribs were short and horizontally placed. Bilateral humerus and femori were extremely short. The vertebral bodies showed flattened (platyspondyly). "Telephone receiver" like curved femora was noted. Cranial sonography showed normal structural of the brain. Mutational analysis confirmed a heterozygous allele p.Tyr373Cys mutation in the FGFR3 gene. With facial features, skeletal abnormalities and mutation analysis the diagnosis of TD type I was made.

Postnatal course was complicated by multiple problems. Subsequent respiratory distress necessitated used CPAP. Chest expansion remained poor possibly due to underlying lung hypoplasia and did not respond well to CPAP. Routine septic work up was performed on day 1 and she was covered empirically with ampicillin and amikacin before definitive diagnosis was reached. Blood culture result was no growth. Antibiotics were continued until 10 days. On the fifth days of hospitalization patient looked icteric and phototherapy was performed for three days. The general condition and respiratory distress did not improve and baby was died on the sixteenth days of hospitalization caused by respiratory distress to a narrow thorax.

\section{References}

[1] Vogt C, Blass HK. Thanatophoric dysplasia: Autopsy findings over a 25-year period. Pediatric and Developmental Pathology. 2013; 16: 160-7.

[2] Wainwright H. Thanatophoric dysplasia: A review. SAMJ. 2016; 106 (6): 50-3.

[3] Hatzaki A, Sifakis S, Apostolopoulou D, Bouzarelou D, Konstantinidou A, Kappou D et al. FGFR3 related skeletal dysplasias diagnosed prenatally by ultrasonography and molecular analysis: Presentation of 17 cases. Am J Med Genet. 2011; 155: 2426-35.

[4] Gopal G, Belavadi GB. Thanatophoric dysplasia in the newborn. J of Evolution of Med and Dent Sci. 2014; 3: 3665-9.

[5] Miller E, Blaser S, Shannon P, Widjaja E. Brain and bone abnormalities of thanatophoric dwarfism. AJR. 2009; 192: 48-51.

[6] Sawai H, Oka K, Ushioda M, Nishimura G, Omori T, Numabe $\mathrm{H}$ et al. National survey of prevalence and prognosis of thanatophoric dysplasia in Japan. Pediatrics International. 2019; 61: 748-53.

[7] Lam ACF, Lam YY, Tong TMF, Chan DKH, Lau WL, Ng DKK et al. Thanatophoric dysplasia type 1 (TD1) without "telephone receivers". HK J Paediatr. 2006; 11: 320-3.

[8] Gulasi S, Atici A, Celik Y. A case of thanatophoric dysplasia type 2: A novel mutation. J Clin Res Pediatr Endocrinol. 2015; 7 (1): 73-6.

[9] Kumar P. Skeletal dysplasias. In: Kumar P, Burton BK, editors. Congenital malformations. Chicago: McGraw-Hill; 2008. p. 307-21.

[10] Sharma M, Jyoti, Jain R, Devendra. Thanatophoric dysplasia: A case report. Journal of Clinical and Diagnostic Research. 2015; 9 (11): 1-3.

[11] Davanageri RS, Shokeen PD, Bannur HB, Patil KP. Thanatophoric dysplasia type I: A rare case report at fetal autopsy. J Lab Physicians 2014; 6: 121-3.

[12] Rai C, Gaikwad HS, Bajaj SK, Gupta R. Thanatophoric dysplasia, an enigmatic dilemma: A case report. Int J Reprod Contracept Obstet Gynecol. 2016; 5 (8): 2856-8.

[13] Jung M, Park SH. Genetically confirmed thanatophoric dysplasia with fibroblast growth factor receptor 3 mutation. Experimental and Molecular Pathology. 2017; 102: 290-5.

[14] Nikkel SM, Major N, King WJ. Growth and development in thanatophoric dysplasia - an update 25 years later. Clinical Case Reports. 2013; 1 (2): 75-8.

[15] Jahan U, Sharma A, Gupta N, Gupta S, Usmani F, Rajput A. Thanatophoric dysplasia: A case report. Int J Reprod Contracept Obstet Gynecol. 2019; 8 (2): 758-61. 